\title{
EFFECT OF SHOCK WAVE REAPPLICATION ON URINARY N-ACETYL- BETA-GLUCOSAMINIDASE IN CANINE KIDNEY
}

\author{
MARCO A.Q.R. FORTES, ADAGMAR ANDRIOLO, VALDEMAR ORTIZ, MIGUEL SROUGI \\ Division of Urology, Paulista School of Medicine, Federal University of São Paulo, UNIFESP, São Paulo, \\ SP, Brazil
}

\begin{abstract}
Objective: Renal tubular damage can be assessed with the aid of urinary dosing of $\mathrm{N}$-acetylbeta-glucosaminidase (NAG) and it is possible to demonstrate a significant correlation between shock wave and damage to renal parenchyma. The objective of this study was to assess the effect of shock wave reapplication over urinary NAG in canine kidney.

Materials and Methods: The authors submitted 10 crossbred dogs to 2 applications of 2000 shock waves in a 24-hour interval in order to assess urinary NAG values after 12, 24, 36 and 48 hours.

Results: Twelve hours following the first shockwave application there was an increase in NAG of $6.47 \pm 5.44 \mathrm{u} / \mathrm{g}$ creatinine ( $\mathrm{p}<0.05$ ). Twelve hours and $24 \mathrm{~h}$ following the second application there was no increase in the urinary enzyme, $-2.56 \pm-7.36 \mathrm{u} / \mathrm{g}$ creatinine and $2.89 \pm-7.27 \mathrm{u} / \mathrm{g}$ creatinine, respectively ( $\mathrm{p}>0.05)$.

Conclusion: Shock wave reapplication with a 24-hour interval did not cause any increase in urinary NAG.
\end{abstract}

Key words: kidney; lithotripsy; high-energy shock waves; acetylglucosaminidase; dogs

Int Braz J Urol. 2004; 30: 148-154

\section{INTRODUCTION}

The first non-invasive method for treating patients with urinary tract lithiasis was introduced by Chaussy in 1980 at the Department of Urology of Munich University (1). Using electrically generated shock waves to fragment stones inside the kidney without any incision, puncture or endoscopic invasion, this first experiment started the extracorporeal shock wave lithotripsy (ESWL) era. Due to its efficacy and safety, ESWL became the method of choice for treating renal stone either as monotherapy or combined with other forms of lithotripsy.

Whatever the generating source is, shock waves can be released in a gradual intensity scale that usually ranges from 14 to $20 \mathrm{KV}$ reaching the target in an area known as focal point, whose diameter varies according to the equipment's specificity.

The safest equipments are those capable of fragmenting the stone using low power in the smallest focal area possible in order to avoid damage to the adjacent renal parenchyma. Though the shock wave is focused on the stone, renal tissue is subjected to trauma as well and, therefore, morphologic and functional changes can occur in this area (2).

The effects of shock waves on the renal parenchyma were studied through imaging scans, histopathological tests and dosage of plasmatic and urinary renal function markers (3-5). Studies using urinary enzymes such as $\mathrm{N}$-acetyl-beta-glucosaminidase (NAG), beta-galactosidase, gamma-glutamyl-transferase and high molecular weight proteins such as 
macroglobulins and calbidin $\mathrm{d} 28 \mathrm{~K}$ assessed renal trauma resulting from shock waves because they are proteins that, under normal conditions, are not filtered by the glomerulus. Following local trauma, they reach the collecting system and can be dosed in urine $(6,7)$. On the other hand, after 2 to 3 days, these markers return to their normal levels indicating that the produced alterations could be transitory and the renal tissue would rapidly recover (8-11).

Thus, due to the prompt recovery from the initial renal trauma, a new lithotripsy session could be performed within a short period interval if the patient required retreatment. However, there is no consensus about which would be the time interval required for retreatment following the first lithotripsy session, with some centers waiting for 3 days, others for one week and others up to one month to repeat lithotripsy $(6,12,13)$.

The intense use of ESWL currently, the lack of knowledge about acute lesions caused by re-treatment within a short time interval and the empirical way by which treatment is conducted, stimulated the development of an experimental model aimed to clarify important doubts in this field of medical knowledge as well as to generate subsidies for new research.

This objective of this work was to assess the effect of shock waves reapplication on urinary $\mathrm{N}$ acetyl-beta-glucosaminidase (NAG) in canine kidney.

\section{MATERIALS AND METHODS}

We used 10 animals that underwent 2 applications of shock waves on the right kidney, with a 24-hour interval between them, and 5 urine collections for determining NAG, according to the study design.

\section{Study Design}

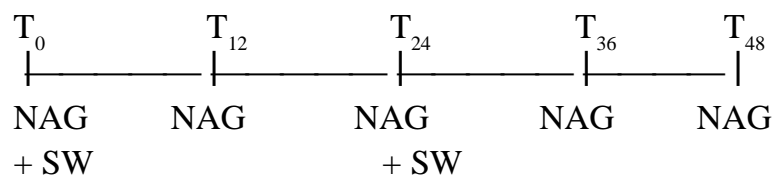

$T=$ time in hours; $S W=$ shock wave $; N A G=N$-acetyl-beta glucosaminidase.
All animals were kept isolated and free from food during 12 hours previously to the session of shock wave application, however they had free access to water.

General anesthesia was induced with intravenous thionembutal $(25 \mathrm{mg} / \mathrm{Kg})$ and maintained with inhalatory pentrane $2 \%$ though endotracheal tube until the end of the session.

A Siemens lithotriptor, model Lithostar Plus Multiline ${ }^{\circledR}$ was used in the experiment. Shock waves are generated by electromagnetic discharges, when the tension peak is induced by fast movements of a membrane under liquid medium. The generator produces a tension ranging from 200 to $300 \mathrm{bar}$. The tension used is chosen according to the variation of the employed voltage, from 13 to $20 \mathrm{KV}$ in intensity. The tension waves propagate through interfaces result in an elliptical-shaped focus, measuring about $11 \times 9$ millimeters. The focus system to be used is obtained by means of acoustic lens and the contact with the animal's skin through a bag containing water and gel.

Previously to the shock wave application, but with the animal already anesthetized, an urine sample was collected in order to perform sedimentoscopy and dosages of creatinine and NAG. Such collection was performed by aseptic supra-pubic puncture. Similarly, urine samples were collected 12 hours after each session and 24 hours after the second session, in the kennel, with the animal under restraint, but without anesthesia.

Thus, during the experiment, 5 urine samples were collected from each animal: 1) The first sample immediately before the first shockwave application $\left.\left(\mathrm{T}_{0}\right) ; 2\right)$ The second sample, in the kennel, 12 hours after the first application $\left(\mathrm{T}_{12}\right) ; 3$ ) The third sample 24 hours after the first application and immediately before the second one $\left(\mathrm{T}_{24}\right)$; 4) The fourth sample 36 hours after the first application and 12 hours after the second one $\left.\left(\mathrm{T}_{36}\right) ; 5\right)$ The fifth sample 48 hours after the first application and 24 hours after the second one $\left(\mathrm{T}_{48}\right)$.

After the first urine collection, tricotomy was performed at the abdominal and lumbar regions, in the contact area between the animal and the water and gel bubble of the shock wave-generating apparatus. The animals were placed over the bubble, in right 
lateral decubitus, and under fluoroscopic guidance, positioned in such a way they would receive the impact of shock waves always on the lower pole of the right kidney. Then, the animals had their paws fixed to the apparatus' table with gauze and 2 crepe tapes were passed, one over the thorax and the other over the lower abdomen in order to prevent its displacement during the procedure.

For fluoroscopic guidance, an intravenous injection of iodinated contrast medium (sodium and meglumine amidotrizoate) was performed at a dosage of $1 \mathrm{mg} / \mathrm{Kg}$ of weight. The injection of contrast medium, in addition to allowing the assessment of the animal's entire urinary tract, guided the focusing of the lower pole of the right kidney, site where the shock wave applications were made.

Every 10 minutes, during the procedure, another dose of contrast medium was injected in order to confirm that there was no change in the focal point due to the animal's movement.

On each application, the animals received 2000 shock wave impulses of $18 \mathrm{KV}$, at a frequency of 100 impulses per minute, during approximately 20 minutes. Upon completing the applications, the animal was taken for the kennel's isolation area, receiving water and proper ration once it was completely recovered from anesthesia.

A part of each collected urine sample was immediately submitted to the laboratory for performing sedimentoscopy. Another part of the urine was stored at $-4^{\circ} \mathrm{C}$ until submission, after a maximum of 30 days, for the laboratory as well, for dosing NAG and creatinine.

In this test, the Na-3-cresolsulphonphthaleinyl$\mathrm{N}$-acetyl-B-D-glucosaminide is hydrolyzed by NAG, releasing 3-cresolsulphonphthalein and sodium salt (3-cresol red), which are photometrically measured at $580 \mathrm{~nm}$. The result for NAG is expressed in $\mathrm{u} / \mathrm{l}$. Urinary creatinine is dosed and the result is expressed in $\mathrm{g} / \mathrm{l}$.

The statistical analysis was performed using the Wilcoxon test due to the paired structure of data in order to compare the variation of NAG values $(\mathrm{u} /$ $\mathrm{g}$ ), which corresponds to the dosage of NAG per gram of creatinine. The significance level was fixed at 0.05 or $5 \%$.

\section{RESULTS}

No change was found in the urine examinations collected before the first shock wave application. All other urine samples collected after the applications presented numberless red cells on sedimentoscopy.

The results from NAG dosages in relation to creatinine and expressed in NAG $\mathrm{u} / \mathrm{g}$ are listed in Table-1.

There was a significant increase in NAG $(\mathrm{u} /$ $\mathrm{g}$ of creatinine) 12 hours after the first shock wave application ( $\mathrm{p}<0.05$ ) (Table-2). After 24 hours such increase was still observed, but it was not regarded as significant $(\mathrm{p}>0.05)$ (Table-2).

The third assessment performed 12 hours after the second shock wave application and 36 hours after the first one did not evidence a significant increase in this enzyme $(\mathrm{p}>0.05)$ (Table-2), similarly to the assessment performed 24 hours after the second application and 48 hours after the first one $(>0.05)$ (Table-2).

\section{DISCUSSION}

The choice of the animal was based in objections made by several authors regarding the discrepancy between the size of the shock wave focus and the kidney size $(5,6,14)$. Studies conducted in small animals such as rats, for example, would have a pro-

Table 1 - Distribution of NAG values expressed in $u / g$ of creatinine in the animals' urine in different moments during the experiment.

\begin{tabular}{lrrrrr} 
Dog & $\mathbf{T}_{\mathbf{0}}$ & $\mathbf{T}_{\mathbf{1 2}}$ & $\mathbf{T}_{\mathbf{2 4}}$ & $\mathbf{T}_{\mathbf{3 6}}$ & \multicolumn{1}{c}{$\mathbf{T}_{\mathbf{4 8}}$} \\
\hline 1 & 5.39 & 17.74 & 10.90 & 2.30 & 11.90 \\
2 & 0.16 & 6.74 & 0.53 & 3.60 & 0.18 \\
3 & 0.61 & 2.17 & 2.08 & 2.45 & 4.00 \\
4 & 4.54 & 4.88 & 5.47 & 4.37 & 0.18 \\
5 & 6.96 & 14.91 & 29.23 & 8.97 & 15.66 \\
6 & 4.80 & 5.22 & 3.11 & 9.21 & 7.59 \\
7 & 3.74 & 12.10 & 2.84 & 2.42 & 22.10 \\
8 & 1.73 & 6.44 & 5.95 & 4.95 & 4.91 \\
9 & 3.10 & 8.05 & 4.21 & 4.84 & 5.78 \\
10 & 3.73 & 21.24 & 4.95 & 0.55 & 0.27 \\
\hline
\end{tabular}


Table 2 - Variation of NAG values (u/g of creatinine) in the animals' urine at different time intervals.

\begin{tabular}{lrrrrrrr}
\hline Dog & \multicolumn{7}{c}{ Time (hours) } \\
& $\mathbf{T}_{\mathbf{1 2}}-\mathbf{T}_{\mathbf{0}}$ & $\mathbf{T}_{\mathbf{2 4}}-\mathbf{T}_{\mathbf{0}}$ & $\mathbf{T}_{\mathbf{2 4}}-\mathbf{T}_{\mathbf{1 2}}$ & $\mathbf{T}_{\mathbf{3 6}}-\mathbf{T}_{\mathbf{1 2}}$ & $\mathbf{T}_{\mathbf{3 6}}-\mathbf{T}_{\mathbf{2 4}}$ & $\mathbf{T}_{\mathbf{4 8}}-\mathbf{T}_{\mathbf{2 4}}$ & $\mathbf{T}_{\mathbf{4 8}}-\mathbf{T}_{\mathbf{3 6}}$ \\
\hline & & & & & & & \\
1 & 12.34 & 5.50 & -6.84 & -15.43 & -8.59 & 1.00 & 9.59 \\
2 & 6.57 & 0.37 & -6.20 & -3.14 & 3.06 & -0.36 & -3.42 \\
3 & 1.56 & 1.47 & -0.09 & 0.29 & 0.38 & 1.92 & 1.54 \\
4 & 0.33 & 0.92 & 0.59 & -0.50 & -1.09 & -5.28 & -4.19 \\
5 & 7.96 & 22.27 & 14.31 & -5.95 & -20.26 & -13.56 & 6.70 \\
6 & 0.41 & -1.70 & -2.11 & 3.99 & 6.10 & 4.48 & -1.62 \\
7 & 8.37 & -0.89 & -9.26 & -9.68 & -0.42 & 19.25 & 10.06 \\
8 & 4.72 & 4.23 & -0.49 & -1.49 & -1.00 & -1.05 & -0.05 \\
9 & 4.94 & 1.11 & -3.83 & -3.20 & 0.63 & 1.57 & 0.94 \\
10 & 17.50 & 1.21 & -16.29 & -20.68 & -4.39 & -4.67 & -0.28 \\
Minimum Value & 0.33 & -1.70 & -16.29 & -20.68 & -20.26 & -4.19 & -4.19 \\
Maximum Value & 17.50 & 22.27 & 14.31 & 3.99 & 6.10 & 19.67 & 19.67 \\
Average & 6.47 & 3.45 & -3.02 & -5.58 & -2.56 & 2.89 & 3.22 \\
Standard-deviation & 5.44 & 6.95 & 7.96 & 7.62 & 7.36 & 7.27 & 7.54 \\
Median & 5.76 & 1.16 & -2.97 & -3.17 & -0.71 & 0.45 & 0.45 \\
p-valor (Wilcoxon & & & & & & & \\
\multicolumn{1}{c}{ test) } & 0.005 & 0.059 & 0.114 & 0.037 & 0.333 & 0.508 & 0.508 \\
\hline
\end{tabular}

portionally larger area of the renal parenchyma that is affected by the shock wave when compared to humans. The area affected by the shock wave in the kidney, referred as focal point, was $11 \times 9 \mathrm{~mm}$ with the lithotriptor employed, corresponding to approximately $10 \%$ of the renal tissue exposed in human kidneys, approximately $25 \%$ in dogs and almost the entire parenchyma in rats. The choice of the number of impulses to be applied was based in previous studies, which concluded that the use of a high number of shock waves is not necessary in order to assess their effects over several tissues (4).

Urine samples were collected by direct supra-pubic puncture in order to avoid contamination of the samples. The option of dosing urinary NAG for assessment of damages caused to the renal parenchyma was because this enzyme is a specific marker for any aggression to the renal parenchyma $(5,8,15)$. Urinary enzymes are considered sensitive markers of renal damage and therefore are more reliable than conventional methods used for investigating renal function. The increase in urinary levels of this enzyme immediately following an aggression to the kidney, in this case by ESWL, and its return to normal levels in a short period makes this enzyme the ideal marker for acute experiments in kidney (16).

The hematuria found in all post-ESWL urine samples reflects the occurrence of renal lesion in an acute form. In this study, hematuria can be explained only by direct tissular action of shock waves, since it was not present previously. It was not possible to conclude if the hematuria was more intense following the first or the second lithotripsy session.

Some clinical assessments did not evidence a significant increase in NAG in patients submitted to lithotripsy $(17,18)$. The majority of clinical studies, however, confirmed such increase $(6,8,9,19-22)$. Experimental studies in rabbits, dogs and rats confirmed such increase as well $(13,23,24)$.

Some factors can produce these contradictory results including the sample size, different equipments with different energy principles, number of impulses 
and focus size, patients' clinical condition (hydration, urinary obstruction and infection), age and gender, location and number of stones, in addition to conditions of urine storage, moment of collection, sensitivity of the dosing method, expression of results and statistical analysis.

Some experimental studies and clinical assessments confirmed the increase in NAG immediately following the shockwave application; most often this increase was detected after 12 hours. However, in some studies, the levels of this enzyme returned to their normal values after 24 hours $(20,21)$.

For this reason we conducted our study performing the first dosage 12 hours after each lithotripsy session, with a significant increase in this enzyme being observed following this period. On the other hand, 24 hours after the first session there was a marked decrease in the release of NAG in the urine enough to make this difference statistically non-significant.

The loss of this lysosomal enzyme immediately following ESWL suggests the presence of corresponding morphologic alterations in the renal tubular system, and the intensity of urinary elimination of the enzyme reflects the severity of the tubular damage (5). Since NAG is an enzyme with high molecular weight $(>70,000)$ it is not filtered by the glomerulus under normal conditions, but when any acute renal damage occurs it reaches the renal tubule's lumen and finally the urine.

The normalization of urinary NAG dosage would mean then the end of acute destruction of tubular cells, but it does not enable us to tell anything about the extension or the disappearance of residual damage to the renal parenchyma.

The behavior of NAG, increasing immediately after the lithotripsy session and decreasing within 1 week was observed in clinical studies and in one experimental study in rats, suggesting that shock waves can cause some acute renal damage that resolves quickly $(7,21,23)$.

In our study only the dosage performed 12 hours after the first shockwave application evidenced a significant increase in NAG. We could expect, also, a new increase in this enzyme 12 hours after the second session, but it did not happen. Not even after 24 hours there was a significant increase in NAG.
Since the release of this enzyme occurs due to the tubular rupture caused by the shock waves and to compression by intra-renal hematomas, it is possible that this phenomenon does not occur with the same intensity following the second lithotripsy session. Since the application area is the same, a second application would not cause, according to our view, increased edema or increased vascular rupture, which would be reflected through an increase in this enzyme.

Confirming this reasoning, other studies did not find also major histological changes, after 2 weeks, in animals undergoing 2 shock wave applications in a 48-hour interval compared with those undergoing only 1 session (25). Thus in another study using high molecular weight markers, among them NAG, considered the shockwave application safe after a 5-day interval (22). In one experimental study with rats it was concluded that shock wave reapplication after 14 days does not increase the alterations in renal morphology caused by the first application (26).

We know that the tubular damage can be quantified. With the aid of urinary NAG dosing it is possible to demonstrate a significant correlation between shock waves and damage to the renal parenchyma. In our study this tubular damage occurred following the first application and remained stable after the second one, performed just 24 hours later.

It is true that many patients submitted to ESWL require a second treatment, but which must be the time period until this reapplication? Our study, though performed in dogs, suggests that any damage produced to the renal parenchyma could be resolved, from a functional point of view, within 24 hours, and the second treatment could occur, thus, only one day later.

Some lithotripsy centers do not perform the second shockwave application shortly after the first one based on the justification that it is necessary to wait for the fragments elimination in order to subsequently decide for another session. If we consider potential complications such as obstruction by stones (steinstrasse), infection and pain, the best option would be to perform the second session as soon as possible so that lower sized fragments would have better chances of being eliminated. 


\section{CONCLUSION}

We concluded that, under the conditions in the present study, high-energy shock waves, when applied to canine kidney produced an increase in urinary NAG 12 hours later, with normalization after 24 hours and when reapplied after 24 hours they did not cause a rise in urinary NAG after 36 and 48 hours.

\section{REFERENCES}

1. Chaussy CG, Brendel W, Schmiedt E: Extracorporeal induced destruction of kidney stones by shock waves. Lancet.1980; 13: 1265-8.

2. Claro JFA: Shock Wave Efects on Renal Growth and Function - Experimental Studies in Rats. Doctoral Thesis, State University of Campinas, 1994.

3. Delius M, Enders G, Xuan Z, Liebich H, Brendel W: Biological effects of shock waves: kidney damage by shock waves in dogs - dose dependence. Ultr Med Biol. 1988; 14: 117-22.

4. Lottmann HB, Archambaud F, Hellal B, Pageyral M, Cendron M: Technetium-dimercapto-succinic acid renal scan in the evaluation of potential long-term renal parenchymal damage associated with extracorporeal shock wave lithotripsy in children. J Urol. 1998; 159: 521-4.

5. Weichert-Jacobsen K, Stockle M, Loch T, Bruske T: Urinary Leakage of tubular enzymes after shock wave lithotripsy. Eur Urol. 1998; 33:104-10.

6. Recker F, Hofmann W, Bex A, Tscholl R: Quantitative determination of urinary marker proteins: a model to detect intrarenal bioeffects after extracorporeal lithotripsy. J Urol. 1992; 148: 1000-6.

7. Erkizan O, Ayder AR, Minareci S, Lekili M, Dincel C: NAG, GGT, creatinine, urea and creatinine clearance before and after ESWL. Int Urol Neprol. 1994; 26: $259-62$.

8. Assimos DG, Boyce HW, Furr EG, Espeland MA, Holmes RP, Harrison LH, et al.: Selective elevation of urinary enzyme levels after extracorporeal shock wave lithotripsy. J Urol. 1989; 142: 687-90.

9. Karlsen SJ, Berg J: Acute changes in kidney function following extracorporeal shock wave lithotripsy for renal stones. Br J Urol. 1991; 67: 241-5.

10. Karlin GS, Schulsinger D, Urivetsky M, Smith AD: Absence of persisting parenchymal damage after extracorporeal shock wave lithotripsy as judged by ex- cretion of renal tubular enzymes. J Urol. 1990; 144: $13-4$.

11. Kirkali Z, Kirkali G, Tahiri Y: The effect of extracorporeal electromagnetic shock waves on renal proximal tubular function. Int Urol Nephrol. 1994; 26: 2557.

12. Drach GW, Dretler S, Fair W, Finlayson B, Gillenwater J, Griffith D, et al.: Report of the United States cooperative study of extracorporeal shock wave lithotripsy. J Urol. 1986; 135: 1127-33.

13. Morris JS, Husmann W, Wilson T, Preminger GM: Temporal effects of shock wave lithotripsy. J Urol. 1991; 145: 881-3.

14. Neisius D, Seitz G, Gebhardt T, Ziegler M: Dose-dependent influence on canine renal morphology after application of extracorporeal shock waves with Wolf Piezolith. J Endourol. 1989; 3: 337-45.

15. Raab WP: Diagnostic value of urinary enzyme determinations. Clin Chem. 1972; 18: 5-25.

16. Sakkas G, Becopoulos T, Karayannis A, Drossos G, Giannopoulou K: Enzymatic evaluation of renal damage caused by different therapeutic procedures for kidney stone disease. Int Urol Nephrol. 1995; 27: 66977.

17. Krongrad A, Saltzman B, Tannenbaum M: Enzymuria after extracorporeal shock wave lithotripsy. J Endourol. 1991; 5: 209-11.

18. Kitada S, Kuramoto H, Kumazawa J, Yamaguchi A, Nakasu H, Hara S: Effects of extracorporeal shock wave lithotripsy on urinary excretion of N-acetyl-betaglucosaminidase. Urol Int. 1989; 44: 35-7.

19. Trinchieri A, Zanetti G, Tombolini P, Mandressi A, Ruoppolo M, Tura M, et al.: Urinary NAG excretion after anesthesia-free extracorporeal lithotripsy of renal Stones: a marker of early tubular damage. Urol Res. 1990; 18: 259-62.

20. Strohmaier WL, Koch J, Balk N, Wilbert DM, Bichler KH: Limitation of shock-wave-induced renal tubular dysfunction by nifedipine. Eur Urol. 1994; 25: 99-104.

21. Uozomi J, Ueda T, Naito S, Ogata N, Yasumatsu T, Koikawa Y, et al.: Clinical significance of urinary enzymes and â2-microglobulin following ESWL. Int Urol Nephrol. 1994; 26: 605-9.

22. Recker F, Bex A, Hofmann W, Uhlschmid G, Tscholl R: Pathogenesis and shock wave rate dependence of intrarenal injury from extracorporeal lithotripsy. J Endourol. 1992; 6: 199-203.

23. Weichert-Jacobsen K, Scheidt M, Kulkens C, Loch T: Morphological correlates of urinary enzyme loss after extracorporeal lithotripsy. Urol Res. 1997; 25: 257-62. 
24. Karlsen SJ, Smevik B, Stenstrom J, Berg KJ: Acute physiological changes in canine kidneys following exposure to extracorporeal shock waves. J Urol. 1990; 143: 1280-3.

25. Fuchs AM, Couson W, Fuchs GJ: Effect of extracorporeally induced high-energy shock waves on the rab- bit kidney and ureter: a morphologic and functional study. J. Endourol. 1988; 2: 341-4.

26. Kira V, Kaufmann OG, Ortiz V, Srougi M: Morphological alterations following of retreatment eletrohiraulic shock waves in rat kidney. J Endourol. 2000; 14 (suppl 1): A6 (BS2-2).

Received: November 24, 2003 Accepted after revision: April 4, 2004

Correspondence address:

Dr. Marco Antônio Fortes

Rua André Grabois, 465

Rio de Janeiro, RJ, 22785-480, Brazil

Fax: + 552134111321

E-mail: fortesmarco@hotmail.com 